\title{
An Approach for Recognizing Modi Lipi using Ostu's Binarization Algorithm and Kohenen Neural Network
}

\author{
Sidra Anam \\ U.P.T.U., Lucknow \\ Pranveer Singh Institute Of Technogoly \\ Kanpur, U.P.(208020)
}

\author{
Saurabh Gupta \\ U.P.T.U., Lucknow \\ Pranveer Singh Institute Of Technogoly \\ Kanpur, U.P.(208020)
}

\begin{abstract}
Character recognition is simple for humans, but it is quite complex to build a software that can recognize characters. A cursive type Indian origin script known as Modi script is used to write various language like Hindi, Gujarati, Kannada, Persian, Tamil and Telugu. We intend to develop such a character recognition system capable to recognize printed and handwritten Modi characters at an electronic speed by scanning the documents. It is a challenging issue to develop a practical Cursive Character Recognition System (CCRS) which can maintain a high recognition accuracy without concerning the quality of input documents. We have developed Modi Script Character Recognizer System (MSCR) using Otsu's Binarization algorithm and Kohonen neural network method. We have considered 22 different characters of Modi script including vowels and consonants to train the system by using Kohonen neural network. The sample data set (training images) of the characters is maintained by taking handwritten samples from different peoples. These images are used to provide training to the system. The obtained results prove the effectiveness of the proposed recognition technique. We have got lower recognition rates of those characters which are similar in shape and structure. Overall, an acceptable character recognition rate, of $72.6 \%$, has been achieved in the case of handwritten characters.
\end{abstract}

\section{General Terms}

Character recognition, Scripts, Binarization, Grayscale, Binary image, Neural Network.

\section{Keywords}

Cursive Character Recognition System (CCRS), Modi Script Character Recognizer System (MSCR), Otsu's Binarization Algorithm, Kohonen Neural Network, Connected Component Analysis (CCA).

\section{INTRODUCTION}

The challenging nature of handwritten recognition and segmentation has attracted the attention of researchers from industry and academic peoples. The processing of Modi script is a difficult task because the Modi handwritten characters are naturally both cursive and unconstrained. Neural network technique is used for training, testing and validating the software system developed to recognize offline handwritten Modi script. But less number of sample data may degrade the performance of that system. Modi script is written in cursive type, hence it creates extra branches in letters. This problem is further complicated as spurious branches and clusters are formed in thinning process. The spurious branches and clusters of small size are easy to remove but bigger size poses problem [1]
License Plate Recognition with the help of Template Matching technique is one of the best research in the field of image to character conversion. Preprocessing stage encompasses an Otsu's method which is nearly new to convert image into binary form, Connected Component Analysis (CCA) technique for license plate detection and segmentation and Template matching is handed down in recognition [2].

Feature extraction technique is also used for handwritten character recognition. This technique is based on recursive sub divisions of the character image so that the resulting sub-images at each iteration have balanced (approximately equal) numbers of foreground pixels. Feature extraction is followed by a two-stage classification scheme based on the level of granularity of the feature extraction technique [3]. For testing purpose of the proposed system, multiple databases are maintained for the effectiveness of the proposed technique.

Neural network based techniques have been widely used for recognition of characters. Also, these techniques are used to develop automatic handwritten character recognition system for English language with high recognition accuracy, minimum training and classification time [4]. Character modeling is performed to find out the character set for recognition; a character metric is developed with respect to the white and black pixels and the network is trained for recognition. An experimental result shows that back propagation network yields recognition accuracy as more than $70 \%$.

\section{REVIEW OF EXISTING TECHNIQUES}

Modi Script Character Recognizer is capable of recognizing printed or handwritten Modi characters. The text is scanned either in the form a printed document or in the form of hand written text. The image is processed to recognize and extract characters from it. Two techniques of recognition such as template matching and neural network have been discussed. When template matching technique is implemented using java, it has been observed that our proposed system works slow as it works at pixel level. For implementing our system MSCR, we have selected neural network training technique as the most efficient to recognize handwritten and printed characters. A specific application of the neural network known as Kohonen neural network is used to recognize Modi script characters.

\subsection{Template Matching Technique}

Template matching is one of the areas of profound interests in character recognition. Using this technique, we compare template image with input image to find similarity between them. It also provides feedback path to verify the correctness. Template is used as pattern for the 
recognition of characters in textual image whereas matching is the process of comparing template with segments. In the process of template matching, comparison is made between segments and predefined set of templates. It is a slow process due to pixel to pixel matching. Template matching with zoning requires extra looping structures thereby improving the efficiency. While implementing this technique, whole image data is stored into an array and divided into different zones. Since the matching process is performed zone by zone, it provides better performance of recognition as compared to simple template matching.

\subsection{Neural Network Technique}

Neural networks have been employed for character recognition with varying degree of success. These networks are also employed for integrating the results of the classifiers by adjusting weights to obtain desired output. The structure of the Kohonen neural network contains only an input and output layer of neurons, without hidden layer. The input to the network is input neurons; each allocated a floating point number (normalized between the range of -1 and 1 ). When the pattern is presented to Kohonen neural network, one single output neuron is chosen as the output neuron. The structure of a typical Kohonen neural network is shown in Figure 2.1. It shows a simple network with only two input neurons i.e. $\mathrm{I}_{1}$ and $\mathrm{I}_{2}$ and two output neurons i.e. $\mathrm{O}_{1}$ and $\mathrm{O}_{2}$. The process of learning of Kohonen Network involves a series of steps which are as follows: Normalizing the Input, Calculating Each Neuron's Output, Mapping to Bipolar, Choosing the Winner, Learning process of Kohonen neural network, Learning Rate, Calculating the Error.

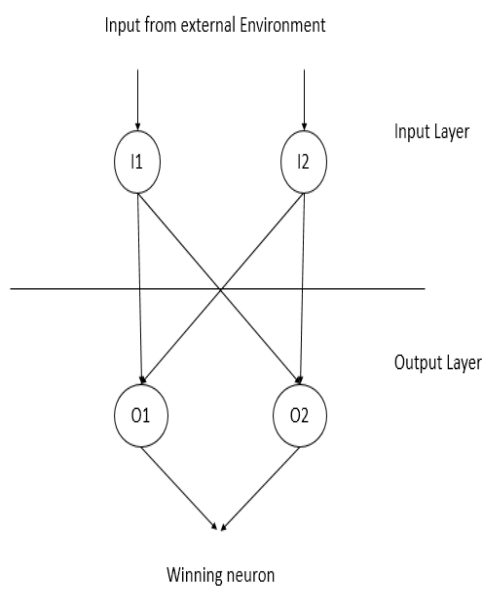

Figure 2.1 Kohonen Neural Network layers

\section{PROPOSED SYSTEM}

MSCR is developed to recognize the characters of Modi script. MSCR accepts input in the form of scanned images, which includes documents written in Modi script. These scanned images are filtered to reduce noise level of the image for enhancing the recognition rate of Modi script characters. It is achieved by preprocessing the image using various transformations, which include Grayscale conversion and Binarization. Preprocessed image is segmented on the basis of pixel intensity, and these segments are extracted into individual characters. MSCR is able to recognize each character of Modi script and convert those characters into Devanagri script. Most of the proposed character recognition systems suffer with some shortfalls such as input and output format restriction, processing time and accuracy. In our system MSCR, we have attempted to put the efforts to overcome some of these limitations. The phases of MSCR system include image acquisition, pre-processing, segmentation and recognition. We discuss them in subsequent sections.

\subsection{Image Acquisition phase}

The recognition system MSCR acquire a scanned image as an input image. The image should have specific formats such as .jpeg, .png, .jpg and .bmp. This image is acquired through a scanner or digital camera or any other suitable digital input device. The acquired image is stored in a buffer and forwarded to the preprocessing phase.

\subsection{Preprocessing Phase}

The purpose of preprocessing is to amend the image data that suppress unwanted distortions. This process also enhances image quality that is required for further processing. There are number of factors that affect the accuracy of character recognition rate such as scanner quality, scan resolution, paper quality, linguistic complexities, etc. Hence, an effective preprocessing algorithm is desirable to overcome these shortcomings. In our system MSCR, preprocessing involves Grayscale image conversion and Binarization techniques that are used to reduce noise caused by the factors mentioned above. These techniques turn the resultant MSCR system more robust. Table 3 shows the processed image before and after preprocessing phase. We discussed these techniques in detail as follows:

\subsubsection{Grayscale Conversion}

Grayscale image contains single intensity value for each pixel, as opposed to the three intensities needed to specify each pixel in a color image. Eq. 3.1 is used to convert the color image into Grayscale image. A single intensity value is calculated for the image. Here R, G and B shows intensity value of Red, Green and Blue colors respectively.

Gray $=0.2126 * \mathrm{R}+0.7152 * \mathrm{G}+0.0722 * \mathrm{~B}$

$$
\text { ... (3.1) }
$$

\subsubsection{Binarization}

Process of converting Grayscale image into bi-level image is known as binarization. Binary image is a digital image that has only two possible values in a single bit $(0$ or 1$)$ for each pixel. Performance of subsequent steps in MSCR depends on the accuracy of the binarization process. The purpose of binarization of image is to extract the text from the image, remove the noise and reduce the size of the image. It has been observed that there exist several algorithms for Binarization that are namely; Global fixed threshold algorithm, Otsu threshold algorithm, Chang's method, Sauvola-Niblack method, Margin error diffusion (Margin ED) and Markov model. We have used Otsu's threshold algorithm for implementing our system MSCR.

\subsection{Segmentation Phase}

Segmentation refers to the process of partitioning a digital image into multiple segments (sets of pixels). A segment is a pattern that resembles one of the characters to which the system MSCR is designed to recognize. This process starts from an initial point in the scanned image of a document. Segment of an image is an area, which distinguishes one character from another and extracts individual attributes of each character such as width, height etc. In actual practice, combinations of the following approaches are used to segment the document, which can be a machine printed or 
hand written. In holistic method, the system seeks to recognize words as a whole, thus avoiding the need to segment into characters. Various hybrid techniques are also available to segment a character. One of these techniques is hit and deflect algorithm. It is used to find an optimal path for cropping a connected component. Another technique known as drop fall algorithm attempts to build a segmentation path by mimicking an object falling or rolling in between the two characters, which make up a connected component. We have derived a method for cropping the image, which segments the image into individual characters more efficiently than the methods mentioned above.

Table 3: Image of Characters and their forms

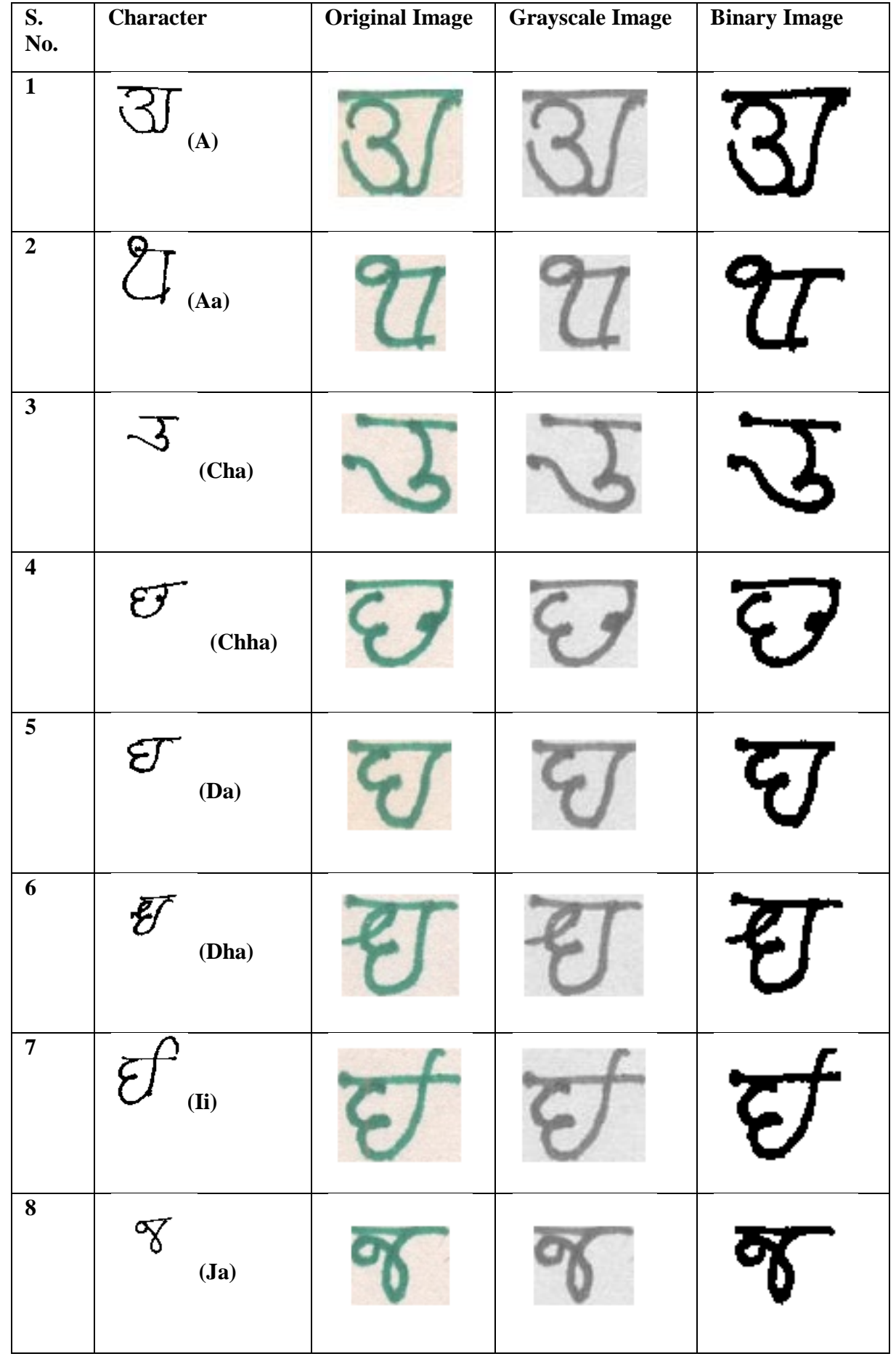




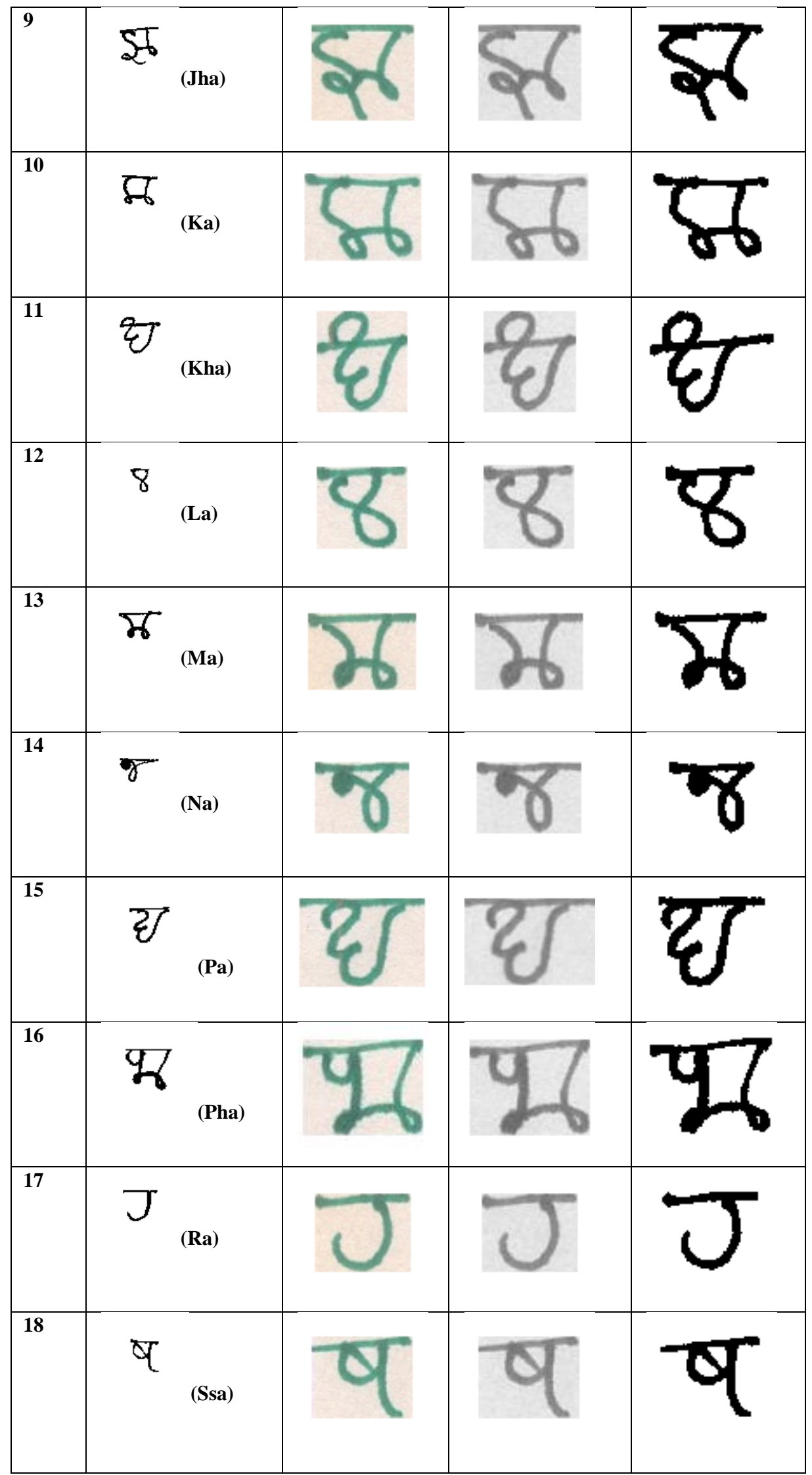




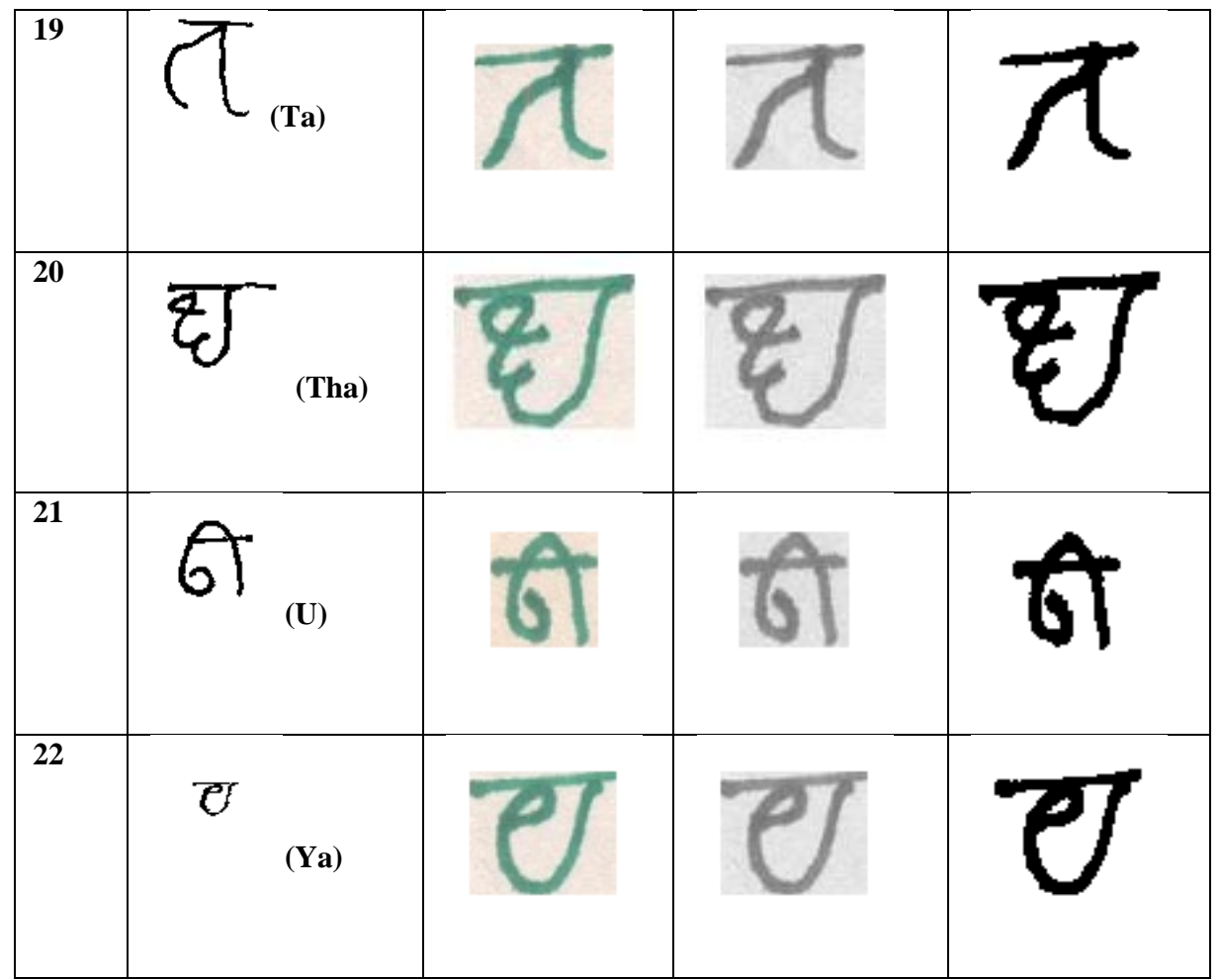

\subsection{Recognition Phase}

Recognition is the process of matching the segmented characters obtained in the previous phase with the data set used for training the network. When a character image matches with the data set image, successful recognition is announced. This can be achieved by using some recognition techniques. One of those techniques is template matching which is an efficient method for recognition of characters with one drawback as it is best for fixed size and fixed font character image only. Hence, we have attained higher recognition rate of handwritten characters as well as printed characters by using Kohonen neural network technique. The program of Kohonen neural network is not immediately ready to recognize letters. This program must be trained from actual drawn letters before it is able to recognize the scanned input. After the training phase our system MSCR is able to recognize Modi script characters.

\section{EXPERIMENTAL RESULS}

The experiment involves large number of data sets collected from different persons. The character set of Modi script is collected in the form of writings from various sources including professional Modi script writers as well as students. These hand written documents are scanned in an image file. These files are presented to MSCR for further recognition process which involves a series of transformation of each image. The preprocessed images are used to train the neural network. For each character, training is provided by using 8 images from the data set. After training the network, testing is performed on our system MSCR by using the test data set. The highest recognition rate attained for handwritten test data set is $94 \%$. The recognition trend for the Modi script characters is provided below in the Table 4 .

Table 4: Experimental Results and Recognition

\begin{tabular}{|c|c|c|c|c|c|}
\hline S. No. & Character & $\begin{array}{l}\text { Total } \\
\text { Number of } \\
\text { Training } \\
\text { Images }\end{array}$ & $\begin{array}{l}\text { Total } \\
\text { Number of } \\
\text { Testing } \\
\text { Images }\end{array}$ & $\begin{array}{l}\text { Total number } \\
\text { of Recognized } \\
\text { Images }\end{array}$ & $\begin{array}{l}\text { Recognition } \\
\text { rate }(\%)\end{array}$ \\
\hline 1 & & 8 & 50 & 37 & 74 \\
\hline 2 & & 8 & 50 & 41 & 82 \\
\hline 3 & $\sqrt{3}(\mathrm{Ca})$ & 8 & 50 & 38 & 76 \\
\hline 4 & & 8 & 50 & 35 & 70 \\
\hline
\end{tabular}




\begin{tabular}{|c|c|c|c|c|c|}
\hline & (Cha) & & & & \\
\hline 5 & $\mathcal{T}_{(\mathrm{Da})}$ & 8 & \begin{tabular}{|l|}
50 \\
\end{tabular} & 32 & 64 \\
\hline 6 & $\overline{\mathscr{V}}$ & 8 & \begin{tabular}{|l|}
50 \\
\end{tabular} & 38 & \begin{tabular}{|l|}
76 \\
\end{tabular} \\
\hline 7 & $\mathcal{E}_{(\mathbf{I i})}$ & 8 & \begin{tabular}{|l|}
50 \\
\end{tabular} & 39 & \begin{tabular}{|l|}
78 \\
\end{tabular} \\
\hline 8 & $g_{(J a)}$ & 8 & \begin{tabular}{|l|}
50 \\
\end{tabular} & 42 & \begin{tabular}{|l|}
84 \\
\end{tabular} \\
\hline 9 & $\begin{array}{l}\text { S6 } \\
\text { (Jha) }\end{array}$ & 8 & 50 & 31 & \begin{tabular}{|l|}
62 \\
\end{tabular} \\
\hline 10 & $\mathscr{L}_{(\mathrm{Ka})}$ & 8 & $\begin{array}{l}50 \\
\end{array}$ & 39 & 78 \\
\hline 11 & ह & 8 & $\mathbf{5 0}$ & 37 & \begin{tabular}{|l|l}
74 \\
\end{tabular} \\
\hline 12 & $\begin{array}{r}8 \\
\text { (La) }\end{array}$ & 8 & $\begin{array}{l}50 \\
\end{array}$ & 35 & \begin{tabular}{|l|}
70 \\
\end{tabular} \\
\hline 13 & $\underset{\text { (Ma) }}{ }$ & 8 & $\mathbf{5 0}$ & 36 & \begin{tabular}{|l|}
72 \\
\end{tabular} \\
\hline 14 & $\begin{array}{c}\gamma \\
(\mathrm{Na})\end{array}$ & 8 & \begin{tabular}{|l|}
50 \\
\end{tabular} & 39 & \begin{tabular}{|l|}
78 \\
\end{tabular} \\
\hline 15 & 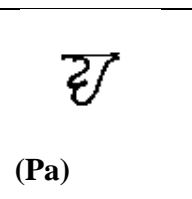 & 8 & 50 & 34 & 68 \\
\hline 16 & $\begin{array}{l}\text { भु } \\
\text { (Pha) }\end{array}$ & 8 & $\mathbf{5 0}$ & 33 & \begin{tabular}{|l|}
66 \\
\end{tabular} \\
\hline 17 & $\begin{array}{c}J \\
(\mathbf{R a})\end{array}$ & 8 & 50 & 47 & \begin{tabular}{|l|}
94 \\
\end{tabular} \\
\hline 18 & $\begin{array}{c}\Phi \\
(\text { Ssa) }\end{array}$ & 8 & $\mathbf{5 0}$ & 37 & \begin{tabular}{|l|}
74 \\
\end{tabular} \\
\hline
\end{tabular}




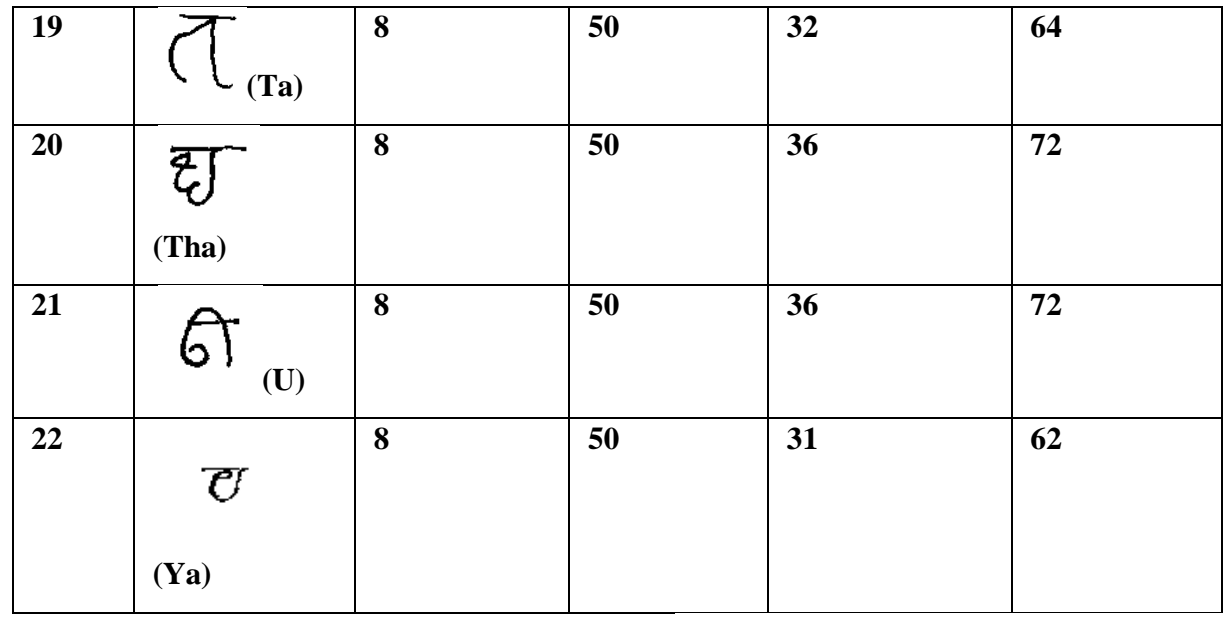

\section{CONCLUSION AND FUTURE SCOPE}

We have developed MSCR using Otsu's Binarization algorithm and Kohonen neural network method. We have considered 22 different characters of Modi script including vowels and consonants to train the system by using Kohonen neural network. The sample data set (training images) of the characters is maintained by taking handwritten samples from different peoples. These images are used to provide training to the system. Here, we have performed numerous character recognition experiments, using the proposed technique. Our recognition system has been tested on various collected datasets and satisfactory results have been obtained. The obtained results prove the effectiveness of the proposed recognition technique. We have got lower recognition rates of those characters which are similar in shape and structure. Overall, an acceptable character recognition rate, of $72.6 \%$, has been achieved in the case of handwritten characters. This work can be extended by incorporating the other scripts available in different languages and increasing the overall acceptable character recognition rate.

\section{REFERENCES}

[1] Clark F. Olson, "On the Speed and Accuracy of Object Recognition When Using Imperfect Grouping”, Proceedings of the International Symposium on Computer Vision, pages 449-454, 1995.

[2] Divya Gilly and Dr. Kumudha raimond, "License Plate Recognition- A Template Matching Method", International Journal of Engineering Research and Applications (IJERA), ISSN: 2248-9622 Vol. 3, Issue 2, March -April 2013, pp.

[3] Pramit Brata Chanda, Santanu Datta, Soham Mukherjee, Subhamoy Goswami and Sritama Bisi, "Comparative Analysis of Offline Character Recognition Using Neural Network Approaches",
Emerging Trends in Computing and Communication, Lecture Notes in Electrical Engineering, Volume 298, 2014, pp 133-140.

[4] A.Ratna Raju, "A Novel Approach For Alphanumeric Character Recognition Using Back Propagation Algorithm", International Journal of Advanced Technology in Engineering and Science, Volume No.02, Issue No. 06, June 2014 ISSN (online): 2348 7550.

[5] M.Y. Savant, Kriti Rakshana, "Marathi MODI Script: Origin, Evolution and Significance", a bi monthly publication of the national mission for manu script, vol 1 no 1, August 2005.

[6] Rakesh A. Ramraje, "History of Modi Script in Maharashrta”, GEIIRJ, vol II Issue 1, June 2013.

[7] Naren Ranadive, "The Origin and Development of Indian Writing System - MODI Script of Maharashtra", http://narendranath.webs.com

[8] A S Ramteke, G S Katkar, "Recognition of Off-line Modi Script : A Structure Similarity Approach", International Journal of ICT and Management, vol. 1, Issue 1, February 2013.

[9] Otsu.N, "A threshold selection method from gray level histograms", IEEE Trans. Systems, Man and Cybernetics, vol.9, pp.62-66, 1979.

[10] Trier.O.D, Jain.A.K and Taxt.J, "Feature extraction methods for character recognition - A survey", Pattern Recognition, vol.29, no.4, pp.641-662, 1996.

[11] Rafael C. Gonzalez, Richard E. Woods, "Digital Image Processing (2nd Edition)”, PHI.

[12] D. N. Besekar, Dr. R. J. Ramteke, "A Chain Code Approach For Recognising Modi Script Numerals", IJOAR, Volume 1, Issue 3, December 2011, ISSN 2249-555X. 